\title{
e-Epidemic Model for the Analysis of Impact of Worms in Computer Network
}

\author{
Kaveri kanchan Kumari \\ Department of Applied Mathematics \\ Cambridge Institute of Technology, Tatisilwai, Ranchi,Jharkhand, India \\ Aditya Kumar Singh \\ Department of Mathematics \\ Government Engineering College, Murbanda, Ramgarh, Jharkhand, India \\ Sahdeo Mahto \\ University Department of Mathematics, Ranchi University, Ranchi, Jharkhand, India
}

\begin{abstract}
With regard to curb the impact and transmission of worms for general form of incidence rate in computer networks, SIQRS(susceptible infectious quarantine recovered susceptible) model has been developed. Basic reproduction number, endemic equilibrium state and their stability are discussed for the general form of incidence rate. With the help of Jacobian matrix and Liapunov function, it has been shown that the system is locally and globally asymptotically stable for the infection free equilibrium state. We have derived an explicit formula for the reproductive number. Numerical methods have been used to solve and simulate the system of differential equations which will help us to understand the attacking behavior of malicious object in computer network, and efficiency of antivirus software.
\end{abstract}

Keywords- Quarantine Epidemic Model; Malicious Objects; Computer Network; Basic Reproduction Number. 2000 Classification- 92D30, 34D23

\section{INTRODUCTION}

Nothing is permanent, everything that exists in nature may change and this vary change gives us a fantastic world of cyber. Over the years, the internet technology has been continuously offering multiple functionalities and facilities. The augmentation of cyber technology has helped in data and information exchange to take at blazing speed which transforms the world into global village. It has made the life easier and world accessible with the touch of button. But everything is not well in cyber world; it is facing several challenges in the form of malicious objects. These malicious objects are worms, virus and Trojan horse. Malicious objects have tremendous influence on computer network. Currently, e-mail has become one of the main factors for the transmission of malicious objects. Transmission of malicious objects in computer network is epidemic in nature and is analogous to biological epidemic diseases. Controlling the malicious objects in computer network has been an increasingly complex issue in recent years. In order to curb the malicious object and analyze the stability of the system, we propose an SIQRS model. In this model, we have used Quarantine class for the very general form of nonlinear incidence rate as a device. Quarantine the word symbolizing a force isolation. The biological world witnessed a radical changes in the last decades with successful implementation of Quarantine to reduce the transmission of human disease such as Leprosy, Plague, Smallpox etc. The same concept has been adopted in the cyber world; the most infected nodes are isolated from the computer network till they get recovered.

Electronic mails and use of secondary devices are the major sources for the transmission of malicious objects in the computer network these days [1]. Anderson and May[2,3] discussed the spreading nature of biological viruses, parasite etc leading to infectious diseases in human population through several epidemic models. The action of malicious objects throughout a network can be studied by using epidemiological models for disease propagation [4-9]. Richard and Mark [10, 11] proposed an improved SEI (Susceptible-Exposed-Infected) model to simulate virus propagation. However, they do not show the length of latency and take into account the impact of anti-virus software. Mishra and Saini [12-14] presented a SEIRS model with latent and temporary immune periods to overcome limitation, which can reveal common worm propagation. Feng and Thieme $[15,16]$ considered very general endemic models that include SEIQR model, with arbitrarily distributed periods of infection including quarantine, and with a general form for the incidence term that includes the three forms. Wa and Feng [17] showed that an epidemic approximation near threshold number [Rq=1] can have a homoclinic bifurcation, so that some perturbation of the original model might also have a homoclinic bifurcation. Several authors studied the global stability of several epidemiological models [18-30]. 
Several authors have studied on bilinear standard incidence rate, but these may require modification, for example the underlying assumption of homogeneous mixing may not be true in cyber world. In this case, the necessary population structure and heterogeneous mixing may be incorporated into a model with a specific form of non-linear transmission. In this work, we assume a general form $\mathrm{f}(\mathrm{S}, \mathrm{I}, \mathrm{N})$ as a non-linear incidence rate constrained with a few e-epidemic feasible conditions. We show that for SIQRS models (a) if basic reproduction

number, that is, $R_{0}>1$ then the endemic equilibrium of the system asymptotically stable, and (b) if $R_{0} \leq{ }_{1}$, then there is no endemic equilibrium state, and the worm infection-free equilibrium state is asymptotically stable. In the next stage we again show that for the instability of the endemic equilibrium state, the incidence rate of $\mathrm{f}(\mathrm{S}, \mathrm{I}, \mathrm{N})$ must be convex with respect to infection I. For global stability, we take incidence rate as a product of two function i.e. $\mathrm{f}(\mathrm{S}, \mathrm{I})=\mathrm{h}(\mathrm{S}) \mathrm{g}(\mathrm{I})$ and then construct a Lyapunov function. We consider a horizontally transmitted infection of worms (transmission from an infective host to a susceptible node) in computer network. We postulate that the incidence rate depends on the variables $\mathrm{S}, \mathrm{I}$, and $\mathrm{N}$ only and is given by a function $\mathrm{f}(\mathrm{S}, \mathrm{I}, \mathrm{N})$.

The function $\mathrm{f}(\mathrm{S}, \mathrm{I}, \mathrm{N})$ must satisfy the conditions $\mathrm{f}(\mathrm{S}, \mathrm{N}, \mathrm{N})=\mathrm{f}(0, \mathrm{I}, \mathrm{N})=0$

and $\frac{\partial f(S, I, N)}{\partial I}>0, \frac{\partial f(S, I, N)}{\partial S}>0$, for all $\mathrm{S}, \mathrm{I}>0$

We also assume that the function $\mathrm{f}(\mathrm{S}, \mathrm{I}, \mathrm{N})$ is concave with respect to the variable $\mathrm{I}$,

that is, $\quad \frac{\partial^{2} f(S, I, N)}{\partial I^{2}} \leq 0$ for all $\mathrm{S}, \mathrm{I}>0$

In the SIQRS model for the infections that does not confer permanent immunity, susceptible nodes go to infectious class, thereafter some nodes remain in the infected class while they are infectious and then move to the recovered class after the run of anti malicious software and other most infected nodes are transferred into the quarantined class while they are infectious and then move to the recovered class after their recovery. The model here have a variable total population size, because they have recruitment into the susceptible class by inclusion of some new nodes and they have crashing of nodes due to the reason other than the attack of malicious Objects.

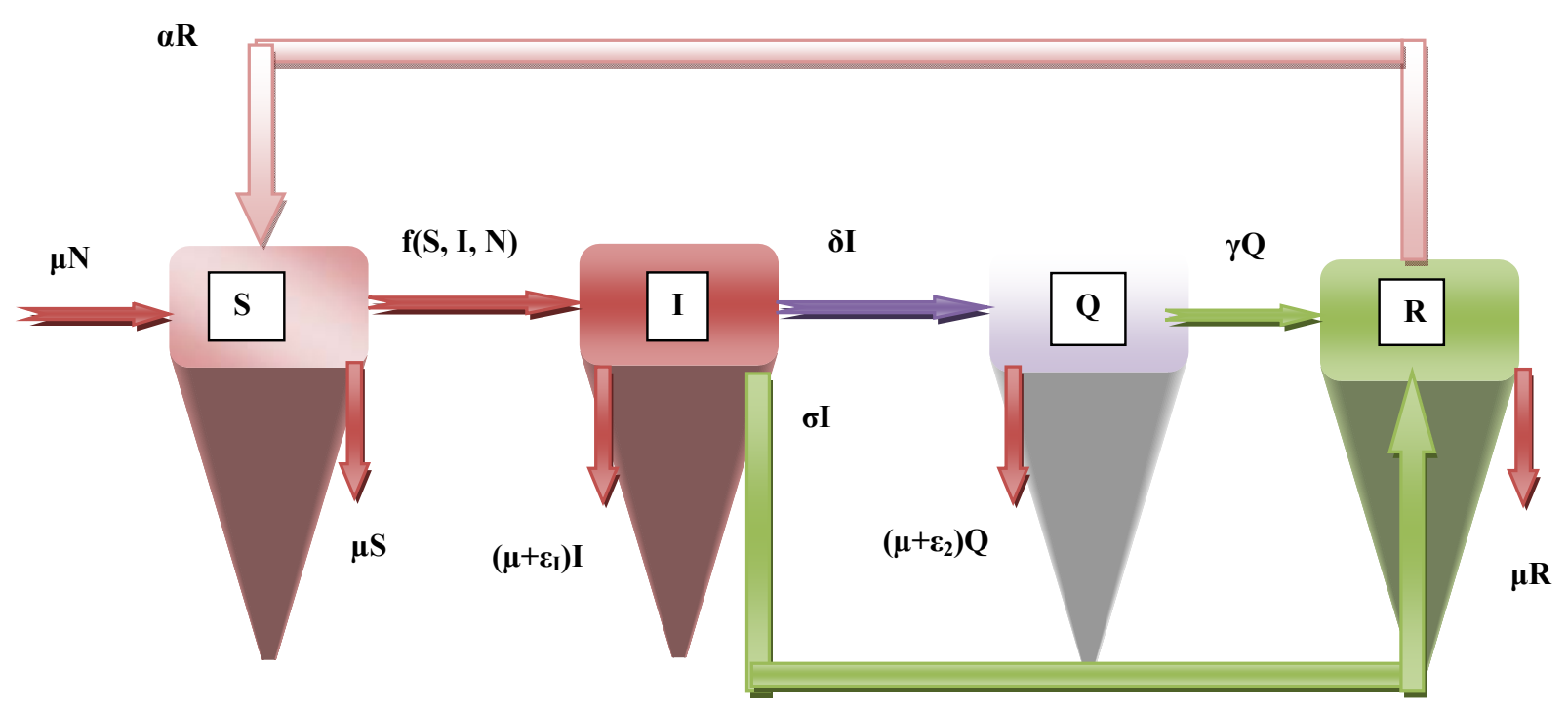

Figure 1. Schematic diagram for the flow of malicious objects in the computer network 


\section{RATE}

\section{MATHEMATICAL FORMULATION FOR THE SIQR MODEL WITH NON LINEAR INCIDENCE}

Let $S(t)$ be the number of susceptible at time $t, I(t)$ be the number of infected nodes, $Q(t)$ be the number of quarantined nodes, $R(t)$ be the recovered nodes after the run of anti malicious software and $N(t)$ be the total population size in time t. The schematic diagram for the flow of malicious objects has been shown in fig 1.

Based on our assumption, we have the following system of differential equations.

$$
\begin{aligned}
& \frac{d S}{d t}=\mu N-f(S, I, N)-\mu S+\alpha R \\
& \frac{d I}{d t}=f(S, I, N)-\left(\delta+\mu+\varepsilon_{1}+\sigma\right) I \\
& \frac{d Q}{d t}=\delta I-\left(\gamma+\mu+\varepsilon_{2}\right) Q \\
& \frac{d R}{d t}=\sigma I+\gamma Q-(\mu+\alpha) R
\end{aligned}
$$

where $\mu$ is the birth rate and death rate due to the reason other than the attack of malicious objects, $\varepsilon_{1}$ be the death rate in infective compartment due to malicious objects, $\varepsilon_{2}$ be the death rate in quarantine class due to malicious objects, $\delta$ is the rate constant which leaves the infective class for quarantine class, $\gamma$ is the rate by which the nodes go from quarantine class into recovered class, $\alpha$ is the rate of transmission of nodes from recovered to susceptible class and $\sigma$ is the rate of transmission of nodes from infectious to recovered class.

We have

$$
\mathrm{N}=\mathrm{S}+\mathrm{I}+\mathrm{Q}+\mathrm{R}
$$

Thus, the reduced system of equations takes form,

$$
\begin{aligned}
& \frac{d S}{d t}=(\mu+\alpha) N-f(S, I, N)-(\mu+\alpha) S+\alpha(I+Q) \\
& \frac{d I}{d t}=f(S, I, N)-\left(\delta+\mu+\varepsilon_{1}+\sigma\right) I \\
& \frac{d Q}{d t}=\delta I-\left(\gamma+\mu+\varepsilon_{2}\right) Q
\end{aligned}
$$

At equilibrium state

$$
\begin{aligned}
& (\mu+\alpha) N=f(S, I, N)+(\mu+\alpha) S+\alpha(I+Q) \\
& f(S, I, N)=\left(\delta+\mu+\varepsilon_{1}+\sigma\right) I \\
& \delta I=\left(\gamma+\mu+\varepsilon_{2}\right) Q
\end{aligned}
$$

At worm infection free equilibrium state, $\mathrm{G}_{0}=\left(\mathrm{S}_{0}, \mathrm{I}_{0}\right)$, where $\mathrm{S}_{0}=\mathrm{N}$ and $\mathrm{I}_{0}=0$.

Apart from the infection free equilibrium state $G_{0}$, the system can have positive endemic equilibrium state $G^{*}$, then we have following lemma: 
Lemma 1-: If the condition $\mathrm{f}(\mathrm{S}, 0, \mathrm{~N})=0=\mathrm{f}(0, \mathrm{I}, \mathrm{N})$ and $\frac{\partial f(S, I, N)}{\partial I}>0, \frac{\partial f(S, I, N)}{\partial S}>0$ for all $\mathrm{S}, \mathrm{I}>0$; $\frac{\partial^{2}}{\partial I^{2}} f(S, I, N) \leq 0$ for all $\mathrm{S}, \mathrm{I}>0$ $\frac{\partial f(S, I, N)}{\partial I}>0 ; \frac{\partial f(S, I, N)}{\partial S}>0, \frac{\partial^{2} f}{\partial f^{2}} \leq 0 \forall S, I>0$,

then at the endemic equilibrium state $I \in\left(0, I^{*}\right) \frac{\partial f\left(s^{*}, I^{*}, N\right)}{\partial I} \leq\left(\delta+\mu+\varepsilon_{1}+\sigma\right)$

where the strict equality hold only if $\frac{\partial^{2} f\left(S^{*}, I, N\right)}{\partial I^{2}}=0$ for all $I \in\left(0, I^{*}\right)$

Proof: - From equation (5) at endemic equilibrium state, $f\left(S^{*}, I^{*}, N\right)=\left(\delta+\mu+\varepsilon_{1}+\sigma\right) I^{*}$

Let, $\bar{f}(I)=f\left(S^{*}, I, N\right)$. Also assume that $\frac{\partial f\left(S^{*}, I^{*}, N\right)}{\partial I}=\frac{d \overrightarrow{f\left(I^{*}\right)}}{d I}>\left(\delta+\mu+\varepsilon_{1}+\sigma\right)$

By the mean value theorem, if $I_{1} \in\left(0, I^{*}\right)$ then

$$
\begin{aligned}
& \frac{d \bar{f}\left(I_{1}\right)}{d I}=\frac{\bar{f}\left(I^{*}\right)-\bar{f}(0)}{I^{*}}=\frac{f\left(S^{*}, I^{*}, N\right)-f\left(S^{*}, 0, N\right)}{I^{*}} \\
& =\frac{f\left(S^{*}, I^{*}, N\right)}{I^{*}}=\frac{\left(\delta+\mu+\varepsilon_{1}+\sigma\right) I^{*}}{I^{*}}=\left(\delta+\mu+\varepsilon_{1}+\sigma\right) .
\end{aligned}
$$

If $I \in\left(I, I^{*}\right)$ then again using the mean value theorem

$$
\begin{aligned}
& \frac{\partial^{2} f\left(S^{*}, I_{0}, N\right)}{\partial I^{2}}=\frac{d^{2} \bar{f}\left(I_{0}\right)}{d I^{2}}=\frac{\frac{d \bar{f}\left(I^{*}\right)}{d I}-\frac{d \bar{f}\left(I_{1}\right)}{d I}}{I^{*}-I_{1}}=\frac{\frac{d \bar{f}\left(I^{*}\right)}{d I}-\left(\delta+\mu+\varepsilon_{1}+\sigma\right)}{I^{*}-I_{1}} \\
& \Rightarrow \frac{\partial^{2} f\left(S^{*}, I_{0}, N\right)}{\partial I^{2}}>0
\end{aligned}
$$

which contradicts the hypothesis of the Lemma and hence

$$
\frac{\partial f\left(S^{*}, I^{*}, N\right)}{\partial I} \leq\left(\delta+\mu+\varepsilon_{1}+\sigma\right) \text {. }
$$

We define the basic reproduction number of the system, if $\frac{d I}{d t}>0$ then malicious object will spread in the system i.e. $f(S, I, N)-\left(\delta+\mu+\varepsilon_{1}+\sigma\right)>0$

$$
f(S, I, N)>\left(\delta+\mu+\varepsilon_{1}+\sigma\right)
$$


$\Rightarrow \frac{1}{\left(\delta+\mu+\varepsilon_{1}+\sigma\right)} \cdot \frac{\partial f(S, I, N)}{\partial I}>1$ then infection will spread in the system and it is denoted by $\mathrm{R}_{0}$

and is called basic reproduction number.

Therefore $\mathrm{R}_{0}=\frac{1}{\left(\delta+\mu+\varepsilon_{1}+\sigma\right)} \cdot \frac{\partial f(S, I, N)}{\partial I}$

Theorem 1

(a) If the incidence rate $\mathrm{f}(\mathrm{S}, \mathrm{I}, \mathrm{N})$ satisfies the conditions (1), (2) and (3) and if $R_{0}>1$, then the endemic equilibrium state $\mathrm{G}^{*}=\left(\mathrm{S}^{*}, \mathrm{I}^{*}\right)$ of the system $(5)$ is asymptotically stable.

(b) If $R_{0} \leq 1$, then there is no endemic equilibrium state, and the worm infection - free equilibrium state is asymptotically stable.

Proof (a): Jacobian of the system (A) is

$$
J=\left[\begin{array}{ccc}
-\frac{\partial f(S, I, N)}{\partial S}-(\mu+\alpha) & -\frac{\partial f}{\partial I}-\alpha & -\alpha \\
\frac{\partial f(S, I, N)}{\partial S} & \frac{\partial f}{\partial I}-\left(\delta+\mu+\varepsilon_{1}+\sigma\right) & 0 \\
0 & \delta & -\left(\gamma+\mu+\varepsilon_{2}\right)
\end{array}\right]
$$

By the Routh-Hurwitz Criterion, the eigen values of the matrix have negative real parts if and only if the inequalities $a_{1}, a_{2}, a_{3}>0$ and $a_{1} a_{2}-a_{3}>0$, hold for the coefficient of the characteristic equation $\lambda^{3}+a_{1} \lambda^{2}+a_{2} \lambda+a_{3}=0$

Characteristic equation of the above matrix

$$
\begin{aligned}
& |J-I \lambda|=\left|\begin{array}{ccc}
-\frac{\partial f(S, I, N)}{\partial S}-(\mu+\alpha)-\lambda & -\frac{\partial f}{\partial I}-\alpha & -\alpha \\
\frac{\partial f(S, I, N)}{\partial S} & \frac{\partial f}{\partial I}-\left(\delta+\mu+\varepsilon_{1}+\sigma\right)-\lambda & 0 \\
0 & \delta & -\left(\gamma+\mu+\varepsilon_{2}\right)-\lambda
\end{array}\right|=0 \\
& -\left(\frac{\partial f}{\partial S}+\mu+\alpha+\lambda\right)\left\{-\left(\gamma+\mu+\varepsilon_{2}+\lambda\right)\left(\frac{\partial f}{\partial I}-\left(\delta+\mu+\varepsilon_{1}+\sigma+\lambda\right)\right\}-\left(\frac{\partial f}{\partial I}+\alpha\right) \frac{\partial f}{\partial S}\left(\gamma+\mu+\varepsilon_{2}+\lambda\right)-\right. \\
& \alpha \frac{\partial f}{\partial S} \delta=0 \\
& \Rightarrow\left(\frac{\partial f}{\partial S}+\mu+\alpha+\lambda\right)\left\{\begin{array}{l}
\gamma \delta+\mu \gamma+\varepsilon_{1} \gamma+\sigma \gamma+\lambda \gamma-\gamma \frac{\partial f}{\partial I}+\mu \delta+\mu^{2}+\mu \varepsilon_{1}+\sigma \mu+\lambda \mu-\mu \frac{\partial f}{\partial I}+\varepsilon_{2} \delta+ \\
\varepsilon_{2} \mu+\varepsilon_{2} \lambda-\varepsilon_{2} \frac{\partial f}{\partial I}+\varepsilon_{2} \sigma+\lambda \delta+\lambda \mu+\lambda \varepsilon_{1}+\lambda \sigma+\lambda^{2}-\lambda \frac{\partial f}{\partial I}
\end{array}\right\}- \\
& \left(\frac{\partial f}{\partial I}+\alpha\right) \frac{\partial f}{\partial I}\left(\gamma+\mu+\varepsilon_{2}\right)-\lambda\left(\frac{\partial f}{\partial I}+\alpha\right) \frac{\partial f}{\partial S}-\alpha \frac{\partial f}{\partial S} \delta=0
\end{aligned}
$$


The above characteristic equation is identical with

$$
\lambda^{3}+a_{1} \lambda^{2}+a_{2} \lambda+a_{3}=0
$$

Coefficient of $\lambda^{2}=\mathrm{a}_{1}=\frac{\partial f}{\partial S}+2 \mu+\alpha+\gamma+\varepsilon_{2}+\sigma>0$

Coefficient of $\lambda=\mathrm{a}_{2}=$

$$
\left.\begin{array}{l}
\left.\frac{\partial f}{\partial S}+\mu+\alpha\right) \gamma+\left(\begin{array}{l}
\gamma \delta+\mu \gamma+\varepsilon_{1} \gamma+\sigma \gamma-\gamma \frac{\partial f}{\partial I}+\delta \mu+\mu^{2}+\varepsilon_{1} \mu+\sigma \mu-\mu \frac{\partial f}{\partial I} \\
+\varepsilon_{2} \delta+\varepsilon_{2} \mu+\varepsilon_{2} \sigma-\varepsilon_{2} \frac{\partial f}{\partial I}
\end{array}\right)-\frac{\partial f}{\partial S}\left(\gamma+\mu+\varepsilon_{2}\right)- \\
\frac{\partial f}{\partial S}\left(\frac{\partial f}{\partial I}+\alpha\right)
\end{array}\right)
$$

$$
\begin{aligned}
& \left(\begin{array}{l}
\frac{\partial f}{\partial S}+\mu+\alpha
\end{array}\right)\left(\begin{array}{l}
\gamma \delta+\mu \gamma+\varepsilon_{1} \gamma+\sigma \gamma-\gamma \frac{\partial f}{\partial I}+\mu \delta+\mu^{2}+\mu \varepsilon_{1}+\sigma \mu-\mu \frac{\partial f}{\partial I} \\
\varepsilon_{2} \delta+\varepsilon_{2} \mu+\varepsilon_{2} \sigma-\varepsilon_{2} \frac{\partial f}{\partial I}-\frac{\partial f}{\partial I} \frac{\partial f}{\partial S}\left(\gamma+\mu+\varepsilon_{2}\right)
\end{array}\right) \\
& -\alpha \frac{\partial f}{\partial S} \delta
\end{aligned}
$$

We obtain, $\mathrm{a}_{1} \mathrm{a}_{2}-\mathrm{a}_{3}>0$.

Therefore, all three roots of the characteristic equation have negative real parts, and hence the endemic equilibrium state $\mathrm{G}^{*}$ is asymptotically stable.

Proof (b):- Since the diagonal elements are real and negative of the above matrix therefore the system is asymptotically stable for the malicious objects free equilibrium state.

\section{GLOBAL PROPERTIES OF SIR AND SEIR SYSTEM}

The majority of the incidence rates can be represented as a product of two functions $f(S, I)=h(S) g(I)$ where $h$ depends only on $\mathrm{S}$ and $\mathrm{g}$ depends only on I. For the incidence rate of the form $\mathrm{h}(\mathrm{S}) \mathrm{g}(\mathrm{I})$ satisfying the condition $\mathrm{h}(0) \mathrm{g}(\mathrm{I})=0=\mathrm{h}(\mathrm{S}) \mathrm{g}(0)$ and $\frac{\partial^{2}}{\partial I^{2}}\{h(S) g(I)\} \leq 0$ for all $\mathrm{S}, \mathrm{I}>0$, direct Lyapunov Method enable us to prove global stability for some models. To construct the Lyapunov function we require an auxiliary function with specific properties. To construct Lyapunov function for SIQRS with incidence rate of the form $h(S) g(I)$, we take $\mathrm{N}=1$, i.e., $\mathrm{S}, \mathrm{I}, \mathrm{Q}$ and $\mathrm{R}$ are the fractions of the susceptibles, the infectives, the quarantined and the recovered in the population and $\mathrm{S}+\mathrm{E}+\mathrm{I}+\mathrm{R}=1$ hold.

The SIQR Model

$$
\begin{aligned}
& \frac{d S}{d t}=\mu N-h(S) g(I)-\mu S \\
& \frac{d I}{d t}=h(S) g(I)-\left(\delta+\mu+\varepsilon_{1}+\sigma\right) I \\
& \frac{d Q}{d t}=\delta I-\left(\gamma+\mu+\varepsilon_{2}\right) Q
\end{aligned}
$$


At equilibrium state,

$$
\begin{aligned}
& \mu N=h(S) g(I)+\mu S \\
& h(S) g(I)=\left(\delta+\mu+\varepsilon_{1}+\sigma\right) I \\
& \delta I=\left(\gamma+\mu+\varepsilon_{2}\right) Q
\end{aligned}
$$

Now, we construct the Lyapunov function to show the global stability of infection free equilibrium state

$$
\begin{gathered}
U(S, I, Q)=S-h\left(S_{0}\right) \int_{0}^{S} \frac{d \tau}{h(\tau)}+c I+B Q \\
\text { Here c }=1
\end{gathered}
$$

We know from total differentiation

$$
\frac{d U}{d t}=\frac{\partial U}{\partial S} \frac{d S}{d t}+\frac{\partial U}{\partial I} \frac{d I}{d t}+\frac{\partial U}{\partial Q} \frac{d Q}{d t}
$$

Now, $\frac{\partial U}{\partial S}=1-h\left(S_{0}\right) \frac{1}{h(S)}: \frac{\partial U}{\partial I}=c ; \frac{\partial U}{\partial Q}=B$

$\therefore(7) \Rightarrow \frac{d U}{d t}=\left(1-\frac{h\left(S_{0}\right)}{h((S)}\right)\{\mu-h(S) g(i)-\mu S\}+c\left\{h(S) g(I)-\left(\delta+\mu+\varepsilon_{1}+\sigma\right) I\right\}+$

$$
B\left\{\delta I-\left(\gamma+\mu+\varepsilon_{2}\right) Q\right\}
$$

$$
\frac{d U}{d t}=\mu(1-S)\left(1-\frac{h\left(S_{0}\right)}{h(S)}\right)+Q\left(\mu+\gamma+\varepsilon_{2}\right)\left\{\frac{h\left(S_{0}\right) g(I)}{Q\left(\gamma+\mu+\varepsilon_{2}\right) B}-1\right\}
$$

Since $\mathrm{h}(\mathrm{S})$ and $\mathrm{g}(\mathrm{I})$ are monotonic functions therefore

$$
\mu(1-S)\left(1-\frac{h\left(S_{0}\right)}{h(s)}\right)<0
$$

And $\left\{\frac{h\left(S_{0}\right) g(I)}{Q\left(\gamma+\mu+\varepsilon_{2}\right) I}-1\right\}<0$.

Therefore $\frac{d U}{d t}<0 \quad$ for all $\mathrm{S}, \mathrm{I}>0$

This implies that the system is globally asymptotically stable for the infection free equilibrium state. 
Table- 1 Parametric values used in simulation

\begin{tabular}{|l|l|}
\hline Parameter & Value \\
\hline $\mathbf{N}$ & $\mathbf{1 7 , 0 0}$ \\
\hline $\mathbf{S}(\mathbf{0})$ & $\mathbf{9 5 0}$ \\
\hline $\mathbf{I}(\mathbf{0})$ & $\mathbf{1 0 0}$ \\
\hline $\mathbf{Q}(\mathbf{0})$ & $\mathbf{2 0 0}$ \\
\hline $\mathbf{R}(\mathbf{0})$ & $\mathbf{0}$ \\
\hline $\boldsymbol{\mu}$ & $\mathbf{0 . 0 5}$ \\
\hline $\boldsymbol{\beta}$ & $\mathbf{0 . 0 0 1}$ \\
\hline $\boldsymbol{\delta}$ & $\mathbf{0 . 0 0 5}$ \\
\hline $\boldsymbol{\varepsilon}_{1}$ & $\mathbf{0 . 2 6}$ \\
\hline $\boldsymbol{\varepsilon}_{2}$ & $\mathbf{0 . 2 7}$ \\
\hline $\boldsymbol{\sigma}$ & $\mathbf{0 . 9 9 2}$ \\
\hline$\gamma$ & $\mathbf{0 . 0 0 8}$ \\
\hline$\alpha$ & 0.007 \\
\hline
\end{tabular}

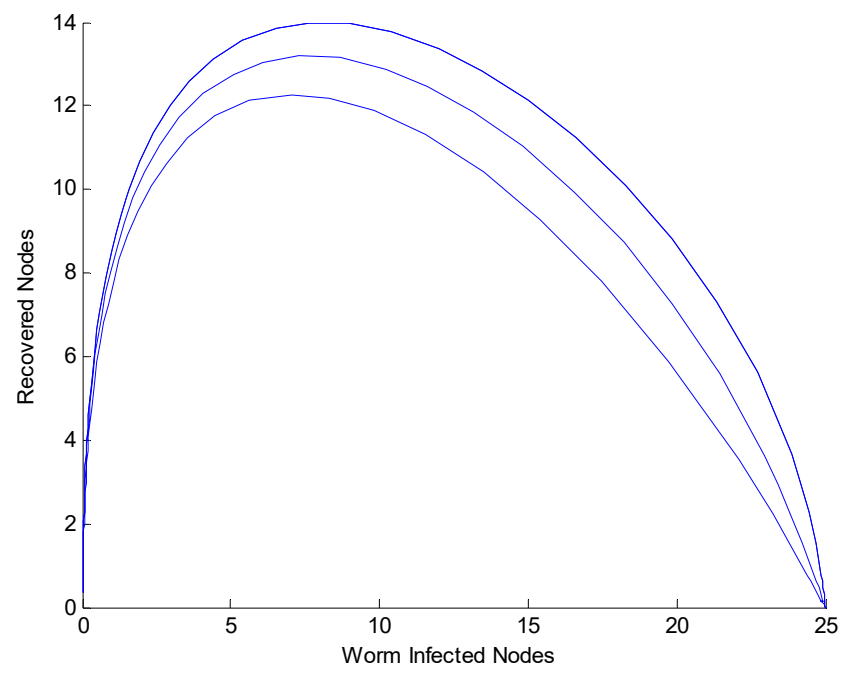

Figure 2. Effect of Worm Infected Nodes $\mathrm{I}_{1}$ on Recovered Nodes R. 


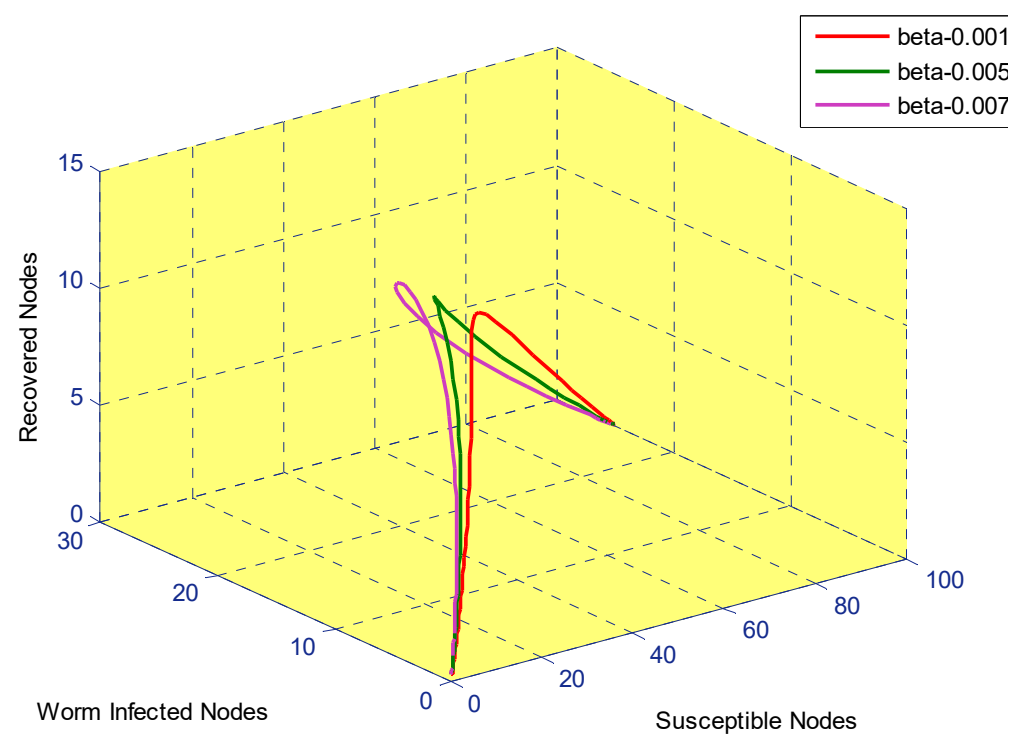

Figure 3. Transformation of nodes from recovered class to susceptible class when attacked by worms

\section{IV.CONCLUSION}

Inspired by the biological compartmental epidemic model, we strived to develop SIQRS model for the transmission of malicious objects with non-linear incidence rate in computer network. We discussed the characteristic of the threshold parameter and keeping into mind the propagating behavior of worms and its self replication characteristics in computer network, we consider a very general form of non-linear incidence rate. The concavity conditions under the constant size assumption are shown to be stable. Local stability with the help of Jacobian matrix for the infection free state if R0 less than or equal to one and endemic equilibrium state if R0 greater than one have been shown and we have also shown the global stability for the infection free equilibrium state. If we take particular form of incidence rate $\beta S I$ instead of general form of incidence rate $f(S, I$, $\mathrm{N}$ ) and simulate the above system of differential equations (4) with the help of Runge-Kutta-Fehlberg fourth fifth order method by using the real parametric values depicted in table 1 . We get the above two graphs which will help us to standard the attacking behavior of worms in computer network. With the analysis of these result, the software company can develop more effective anti malicious software.

\section{REFERENCES}

[1] M. N. J. Newman, Stephanie Forrest, Justin Balthrop, Email network and the spread of computer viruses, Rev. E 66(2002) 035101035104.

[2] R.M. Anderson, R. M. May, Population biology of infection disease,Nature 180 (1999) 361-367.

[3] R. M. Anderson, R. M. May, Infection Disease of humans; Dynamics and control, Oxford University Press, Oxford 1992.

[4] E. Gelenbe, Dealing with software viruses: a biological paradigm, Inform. Security Technical Rep, 12(4) (2007) 242-250.

[5] Erol Gelenbe, Keeping viruses under control, in: Computer and Information science - ISCIS 2005, 20th International symposium, vol. 3733,Lecture Notes in Computer Science, Springer, October 2005.

[6] Erol Gelenbe, Varol Kapton, Yu Wang, Biological metaphor for agent behavior, in;Computer Science, Springer-verlog, October 2004, pp. 667-675.

[7] Bimal Kumar Mishra, Neha Keshri, Mathematical model on the transmission of worms in wireless sensor network, Applied MathematicalModelling, Volume37, Issue 6, March2013, pp. 4103-4111.

[8] J.R.C. Piqueira, B.F. Navarro, L.H.A. Monteiro, Epidemiological models applied to virus in computer networks, J. Comput.Sci.,1(1)(2005) 31-34.

[9] S. Forest, S. Hofmeyr, A.Somayaji, T. Longstaff, Self-nonself discrimination in a computer, in :proceedings of IEEE, Symposium on computer security and privacy, 1994,pp.202-212.

[10] W. T. Richard, J. C. Mark, Modeling virus propagation in peer-to-peer networks, in: IEEE International conference on information, Communications and signal processing (ICICS 2005),pp.981-985.

[11] Bimal Kumar Mishra, D. K. Saini, SEIRS epidemic model with delay for transmission of malicious objects in computer network, Appl.Math Computation,188(2) (2007) 1476-1482.

[12] Bimal Kumar Mishra, Dinesh Saini, Mathematical models on computer virus, Appl. Math. Computation, 187(2) (2007)929-936.

[13] Hemraj Saini, Bimal Kumar Mishra, T. C. Panda, Computing the Spreading power of a business portal to propagate the malicious information in the network, IJWP3(2): 14-22(2011).

[14] Z. Feng, H. R. Thieme, Recurrent outbreaks of childhood disease revisited: The impact of isolation, Math. Biosci, 128 (1995) 93.

[15] Z. Feng, H. R. Thieme, Endemic models with arbitrarily distributed periods of infection ,I:General theory, SIAM J. Appl. Math 61(2000) 803. 
[16] Z. Feng, H. R. Thieme, Endemic models with arbitrarily distributed periods of infection, II: Fast disease dynamics and permanent recovery, SIAM J. Appl. Math. 61 (2000) 983.

[17] L. I. Wu, Z .Feng, Homoclinic bifurcation in an SIQR model for the childhood disease, J. Differ. Equation, 168 (2000) 150

[18] D Green Halgh, Holf bifurcation in epidemic models with a latent period and non permanent immunity, Math. Computer model, 25(1997) 85-107.

[19] H. W. Hethcote, H. W. Stech, P.van dan Driessche, Periodicity and Stability in endemic models: a survey, in : K. L. Cook(Ed.) Differential Equations and Applications in Ecology, Epidemics and Population problems, Academic Press, New York, 1981 PP. 65-85.

[20] K. L. Cook, P.van den Drissche, Analysis of SEIRS epidemic model with two delays, J. Math. Bio, 35(1996) 240-260.

[21] M. Y. L. J.R.Graff, L. C. Wang, J. Karsai, Global Dynamics of a SEIR model with a varying total population size, Math. Biosci, 160 (1999) 191-213.

[22] M.Y. Li, J.S Muldowney, Global Stability for the SEIR model in epidemiology, Math. Biosci, 125(1995) 155-164.

[23] M. Y .Li ,L. Wang, Global stability in some SEIR epidemic models ; C.C Chavez, S .Blower, P. Vanden Driessche, D. Kirscner, A.A Yakhdu (Eds), Mathematical approaches for emerging and reemerging in infectious diseases ;Models, method, Theory, Vol.126,Springer 2002 pp.295-312.

[24] Y .Michel, H. Smith, L .Wang, Global dynamics of SEIR epidemic model with vertical transmission, SIAM journal of Applied Mathematics, 62(1) (2001) 58-69.

[25] J .K. Hale, Ordinary differential equations, second ed., Krieger, Basel, 1980.

[26] H. Hethcote, Mzhein, L. Shengbing, Effects of quarantine in six epidemic models for the infectious diseases, Math. Biosci. 180(2002) 141-160.

[27] B. K. Mishra, N Jha, SEIQRS model for the transmission of malicious object in computer network, Applied Mathematical. Modeling, (2009), 34(2010), 710-715

[28] Bimal Kumar Mishra, Samir Kumar Pandey, Fuzzy epidemic model for the transmission of worms in Computer network, Nonlinear Analysis: Real World Applications, 11 (2010) 433-4341.

[29] Bimal Kumar Mishra, Kaushik Halder, e-Epidemic models on the attack and defense of malicious objects in networks. Theories and Simulation of Complex Social System, 2014, 117-143.

[30] Bimal Kumar Mishra, Gholam Mursalin Ansari, Differential epidemic model of virus and worms in computer network, I. J. Network security 14(3): 149-155(2012). 